\title{
Artrodesis metatarsofalángica del hallux: comparación retrospectiva entre placa bloqueada y tornillos canulados
}

\author{
Hallux metatarsophalangeal arthrodesis: retrospective comparison \\ between blocked plate and cannulated screws
Martínez-García A,* Arvinius C,* Checa-Betegón P,* del Pozo-Martin R,* Manrique-Gamo E,* Galeote-Rodríguez JE*

Hospital Clínico San Carlos, Madrid, España.

\begin{abstract}
RESUMEN. Introducción: Existen múltiples métodos e implantes utilizados para la artrodesis metatarsofalángica del hallux sin evidencia a favor de uno u otro en la bibliografía. El objetivo fue comparar los resultados clínicos y radiológicos de dos métodos e implantes utilizados en nuestro centro. Material y métodos: Revisión retrospectiva de 37 pies entre 2013 y 2017 (22 pies mediante placa y 15 mediante tornillos canulados). Se recogieron variables clínicas y radiológicas y se aplicaron las escalas AOFAS y Manchester-Oxford. Se estudiaron las variables descriptivas y analíticamente mediante el programa SPSS v15. Resultados: El grupo intervenido mediante placa lo compuso $77 \%$ de mujeres, con una edad media de 65 años y una distribución por diagnósticos de hallux valgus (HV) severo (36\%), recidiva de hallux valgus (RHV) (36\%) y hallux rigidus (HR) (28\%), frente a $80 \%$ de mujeres, con media de 68 años y HVS (34\%), RHV (46\%) y HR (20\%) en el grupo de tornillos canulados. No se encontró diferencias significativas en la corrección de los ángulos para HVS o RHV. La tasa de seudoartrosis dolorosa fue de $13 \%$ en ambos y la reintervención fue de $18 \%$ en el grupo de placas y $26 \%$ en el grupo de tornillos. Tanto la escala AOFAS como la M-O fueron mejores en el grupo de placa 63.8 versus 52.6 ( $\mathrm{p}$ $=0.07)$; 30.1 versus $41.0(\mathrm{p}=0.10)$; así como la satisfacción del paciente 86 versus $66 \%$ ( $p>0.05)$ y el dolor postoperatorio 3.68 versus $5.58(\mathrm{p}>0.05)$. Conclusión: Son grupos pequeños de estudio, sin aleatorización de implantes, ambas opciones son funcionales sin poder encontrar una preferente.
\end{abstract}

Palabras clave: Artrodesis, pie, placa, tornillos, tratamiento.
ABSTRACT. Introduction: There are multiple methods and implants used for the metatarsophalangeal arthrodesis of hallux without evidence in favor of one or the other in the bibliography. The goal was to compare the clinical and radiological results of 2 methods and implants used in our center. Material and methods: Retrospective review of 37 feet between 2013 and 2017 (22 feet by plate and 15 by cannulated screws). Clinical and radiological variables were collected and the AOFAS and Manchester-Oxford scales were applied. Variables were studied descriptively and analytically through the SPSSv15 program. Results: The group intervened by plate was composed of $77 \%$ of women, with an average age of 65 years and a distribution by diagnosis of severe hallux valgus (HV) (36\%), hallux valgus relapse (HVR) (36\%) and hallux rigidus (HR) (28\%), compared to $80 \%$ of women, 68 years old and HV (34\%), HVR (46\%) HR (20\%) in the group of cannulated screws. No significant differences were found in the correction of angles for HV or HVR. The rate of painful pseudoarthrosis was $13 \%$ in both and the re-intervention was $18 \%$ in the plate group and $26 \%$ in the screw group. Both the AOFAS and $\mathrm{M}-\mathrm{O}$ scales were better in the plate group 63.8 vs $52.6(\mathrm{p}=0.07)$; 30.1 vs $41.0(\mathrm{p}=0.10)$; as well as patient satisfaction $86 \%$ vs $66 \%$ ( $p>0.05)$ and postoperative pain 3.68 vs 5.58 ( $p>0.05$ ). Conclusion: Small study groups, not implant randomization, both options are functional without being able to find a preferred one.

Keywords: Arthrodesis, foot, plate, screws, treatment.

\section{Nivel de evidencia: IV}

* Médico Ortopedista.

Dirección para correspondencia:

Álvaro Martínez García

Calle de Bretón de los Herreros 56, 2d, 28003, Madrid, España.

E-mail: alvarommg1990@gmail.com 


\section{Introducción}

La artrodesis metatarsofalángica (MTF) del hallux es un procedimiento de indicación creciente, ya que se han publicado resultados clínicos satisfactorios, baja tasa de complicaciones y altas tasas de consolidación en todas sus indicaciones principales: hallux valgus severo, recidiva de hallux valgus y hallux rigidus. ${ }^{1}$ Existen múltiples implantes válidos para esta intervención: tornillos canulados de compresión, placas bloqueadas, placas no bloqueadas, grapas, agujas, combinaciones de los anteriores y técnicas percutáneas. ${ }^{2,3}$ Pero a pesar de toda la evidencia clínica actual, no se ha demostrado superioridad a nivel de resultados clínicos de ninguno de ellos. ${ }^{4}$ El objetivo principal de la artrodesis MTF es conseguir una fijación rígida y una consolidación lo más rápida posible en la mejor posición funcional. ${ }^{5}$

A nivel radiológico y biomecánico algunos implantes parecen demostrar superioridad frente a otros; sin embargo, esto no se suele traducir en resultados clínicos relevantes en los estudios; a esto se suman resultados dispares en los múltiples estudios comparativos que se encuentran en la literatura. ${ }^{4,5,6,7}$ El objetivo de nuestro estudio fue comparar los resultados clínicos y radiológicos de dos métodos de fijación realizados simultáneamente en nuestro centro en la artrodesis MTF: placa bloqueada y tornillos canulados cruzados.

\section{Material y métodos}

Se realizó un estudio retrospectivo de pacientes intervenidos de artrodesis MTF realizados en el Hospital Clínico San Carlos de Madrid entre Enero de 2013 y Octubre de 2017. Se incluyeron en el estudio 33 pacientes, cuatro intervenidos bilateralmente de artrodesis MTF del primer dedo entre 2013 y Octubre de 2017. Las tres indicaciones por la que estos pacientes fueron intervenidos eran: hallux valgus severo, recidiva de hallux valgus y hallux rigidus. Se excluyeron aquéllos que no tenían un mínimo de seis meses de seguimiento. Obtuvimos dos grupos en función de la técnica quirúrgica que se utilizó: 22 pies intervenidos mediante placa bloqueada (Ortholoc ${ }^{\circledR}$ Wright. Memphis TN) y 15 pies intervenidos mediante tornillos de compresión cruzados (Acutrak $^{\circledR}$, Acumed, Hillsboro OR).

Todos los pacientes fueron intervenidos por el mismo equipo de la Unidad de Pie de nuestro centro. Los pacientes fueron colocados en decúbito supino y bajo isquemia del miembro inferior. En el caso de las placas bloqueadas se procedía a un abordaje dorsal de la articulación, fresado de las superficies articulares mediante fresas cónicas y cóncavas, colocación de la placa y compresión dinámica del foco y posterior colocación de tornillos bloqueados a la placa (Figura 1). Para los tornillos canulados se realizó un abordaje dorso medial, resección mediante sierra o fresado de las superficies articulares y posterior colocación de un tornillo de compresión desde el primer metatarsiano a la falange y otro de la falange al primer metatarsiano. El postoperatorio de los pacientes fue común en ambos procedimientos:

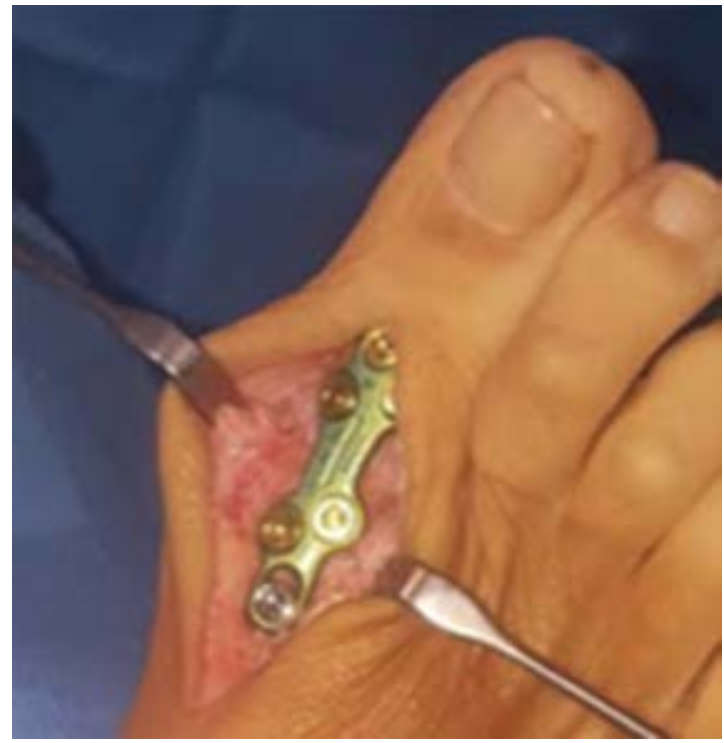

Figura 1: Artrodesis metatarsofalángica mediante placa bloqueada.

consistió en carga inmediata asistida por zapato de tacón invertido durante seis semanas.

Se analizaron las siguientes variables: edad, sexo, antecedentes de cirugías previas, procedimientos asociados y tiempo total del seguimiento hasta la inclusión en el estudio. Asimismo, se evaluaron las complicaciones y reintervenciones que ocurrieron durante el seguimiento. Se revisaron las radiografías (dorsoplantar y lateral en carga) preoperatorias $\mathrm{y}$ al primer, tercer, quinto y sexto mes postoperatorio. Se evaluó el tiempo hasta conseguir consolidación radiológica que se estableció como el mes postoperatorio en el que en la radiografía se apreciaba al menos un puente óseo en ambas proyecciones; además, se evaluaron los ángulos intermetatarsal y del hallux tanto pre- como postoperatoriamente. Se realizó un estudio funcional mediante la escala de Manchester-Oxford, la escala AOFAS del antepié modificada para la valoración de la artrodesis metatarsofalángica y se le preguntó acerca de la satisfacción con la cirugía y el dolor postoperatorio que recordaban del 1 al 10 .

El análisis estadístico fue realizado mediante el programa informático SPSS versión 15. Las pruebas estadísticas de comparación entre variables fueron no paramétricas.

\section{Resultados}

Se obtuvo una edad media de 65 años en el grupo 1 (placas bloqueadas) frente a 67 años en el grupo 2 (tornillos canulados), $77 \%$ fueron mujeres en el grupo 1 frente a $80 \%$ en el grupo 2.

La distribución por diagnósticos no presentó diferencias estadísticamente significativas con lo que eran comparables en ambos grupos; sin embargo, el tiempo de seguimiento fue diferente, estadísticamente significativo (Tabla 1). Los resultados radiológicos fueron similares en ambos grupos (Tabla 2). 
Ambos grupos tuvieron un porcentaje de $13 \%$ de seudoartrosis dolorosa que requirió reintervención quirúrgica, el grupo 2 tuvo una tasa de reintervención por molestias al material de $26 \%$ frente a $18 \%$ en el grupo $1(p=0.31)$, siendo el tiempo medio hasta reintervención de 10 meses en el grupo 1 frente 13 meses en el grupo 2.

Los resultados clínicos que obtuvimos fueron de 63.2 puntos en la escala AOFAS modificada en el grupo 1 frente a 52.6 puntos en el grupo $2(p=0.07)$ y en cuanto a la escala Manchester-Oxford fue de 30.1 puntos en el grupo 1 frente a 41 en el grupo $2(p=0.10)$. Ochenta y seis por ciento de pacientes en el grupo 1 se declaraba satisfechos frente a $66 \%$ en el grupo 2 y el dolor que recordaban en el postoperatorio era de 3.7 (grupo 1) frente a 5.4 (grupo 2) p = 0.13.

\section{Discusión}

La artrodesis metatarsofalángica ha demostrado ampliamente en la literatura ser una buena opción quirúrgica para el tratamiento del hallux valgus severo, la recidiva de hallux valgus y el hallux rigidus; no obstante, la elección del implante presenta muchas más controversias sin encontrarse en la literatura clara evidencia clínica y presentándose resultados a veces contradictorios. ${ }^{1,2,4}$ Los estudios en cadáver y laboratorio parecen haber demostrado la superioridad mecánica de la placa bloqueada frente a los sistemas de exclusiva compresión como los tornillos canulados, las placas dinámicas y las grapas de memoria. ${ }^{3}$ Existen largas series descriptivas de pacientes publicadas en la literatura en las que se ha utilizado un método de fijación, en general, éstas obtienen buenos resultados. Asif y colaboradores obtuvieron una tasa de seudoartrosis de $6.6 \%$ con fijación con tornillos canulados cruzados en una serie de 166 pies; asimismo, Donegan y colegas en una serie de 262 pies fijados con tornillos canulados cruzados obtuvieron $96.18 \%$ de tasa de fusión, con un AOFAS medio de 52 y una satisfacción de $90.72 \% .{ }^{8,9}$ Estos resultados, comparables con los nuestros, objetivan resultados muy satisfactorios de los tornillos canulados aunque sin compararlos con otros métodos de fijación. Las placas bloqueadas son de uso más reciente y por ello no existen grandes series de pacientes y los estudios comparativos, a pesar de avalar buenos resultados, no encuentran diferencias significativas comparando con otros métodos de fijación.

En nuestro estudio comparativo tanto la tasa de fusión (81 versus 69\% a los seis meses) como el tiempo hasta la consolidación fueron mejores en el grupo de placas blo-

Tabla 1: Tiempo de seguimiento y distribución por diagnósticos.

\begin{tabular}{|lcc|}
\hline & $\begin{array}{c}\text { Placa } \\
\text { bloqueada (\%) }\end{array}$ & $\begin{array}{c}\text { Tornillos } \\
\text { canulados (\%) }\end{array}$ \\
\hline Tiempo del Seguimiento Medio & 12 meses & 50 meses \\
Hallux valgus severo (HVS) & 36 & 34 \\
Recidiva de hallux valgus (RHV) & 36 & 46 \\
Hallux rigidus (HR) & 28 & 20 \\
\hline
\end{tabular}

\begin{tabular}{|c|c|c|c|}
\hline & Placa & Tornillos & $\mathrm{p}$ \\
\hline $\begin{array}{l}\text { Tiempo hasta } \\
\text { consolidación radiológica }\end{array}$ & 2.81 meses & 4.73 meses & 0.14 \\
\hline \multicolumn{4}{|l|}{ Ángulo IM: } \\
\hline Pre- & $10.6^{\circ}$ & $13.4^{\circ}$ & 0.35 \\
\hline Post- & $8.6^{\circ}$ & $11.0^{\circ}$ & 0.42 \\
\hline Diferencia (HV y RHV) & $2.0^{\circ}$ & $2.4^{\circ}$ & 0.54 \\
\hline \multicolumn{4}{|l|}{ Ángulo del hallux } \\
\hline Pre- & $40.9^{\circ}$ & $46.9^{\circ}$ & 0.26 \\
\hline Post- & $19.2^{\circ}$ & $29.6^{\circ}$ & 0.21 \\
\hline Diferencia (HV y RHV) & $21.7^{\circ}$ & $17.3^{\circ}$ & 0.33 \\
\hline $\begin{array}{l}\text { Consolidación Rx a los seis } \\
\text { meses (\%) }\end{array}$ & 81.00 & 69.00 & 0.20 \\
\hline
\end{tabular}

queadas, esto en concordancia con otros estudios comparativos. Denning y su grupo equipararon retrospectivamente placas bloqueadas versus tornillos en una serie de 60 pacientes obteniendo $92 \%$ de tasa de fusión frente a $85 \%$ en el grupo de tornillos canulados con resultados clínicos buenos en ambos grupos; Classen y su equipo de investigación compararon retrospectivamente 60 pacientes intervenidos mediante placa no bloqueada, bloqueada y tornillos canulados, encontrando mayor tasa de seudoartrosis (20\%) en los tornillos canulados frente a la placa bloqueada (5\%) sin mostrar diferencias en la evaluación clínica. ${ }^{6,10}$ James y colegas a su vez contrastaron placas bloqueadas frente a no bloqueadas consiguiendo mayor tasa de consolidación en las bloqueadas sin comparar resultados clínicos. ${ }^{11}$

Pocos estudios comparan mediante mediciones radiológicas las diferencias en la corrección de los ángulos entre los distintos dispositivos. En nuestro estudio no hubo diferencias significativas entre los dos implantes, pero observamos que la artrodesis metatarsofalángica modifica el ángulo intermetatarsal, al igual que en otros estudios. ${ }^{12}$ En la literatura actual los resultados de consolidación radiológicos tanto de placas bloqueadas como de tornillos canulados son buenos y comparables en las series más amplias que existen, esto ha sido demostrado incluso mediante tomografía computarizada (TC); sin embargo, no hablan de superioridad clínica, ya que pese a que en la literatura parece haber mayores tasas de consolidación con las placas bloqueadas esto no se traduce en mejores resultados clínicos. ${ }^{1,10,13,14}$

En nuestro estudio se encontró una superioridad en la consolidación radiológica en las placas bloqueadas y una superioridad en las tasas de satisfacción (86 versus 66\%) y en las escalas clínicas (AOFAS y Manchester-Oxford). Esta superioridad mecánica y radiológica que parecen tener las placas bloqueadas puede justificar la superioridad clínica que obtuvimos en nuestra serie, cercana a la significación estadística. Asimismo, en nuestra serie las tasas de reintervención y complicaciones fueron similares en los dos grupos sin encontrarse diferencias.

Nuestro estudio está compuesto por dos grupos comparables operados en el mismo centro y por los mismos ciru- 
janos (tres cirujanos de pie y tobillo) en un lapso de tiempo pequeño y simultáneo (2013-2017). Debido a que en nuestro centro clásicamente se optaba por los tornillos canulados y que durante estos años han sido reemplazados por la placa bloqueada, los tiempos de seguimiento son diferentes; no obstante, en el momento de la evaluación la mayoría de pacientes en ambos grupos presentaba consolidación y un estado clínico estable. Nuestros resultados radiológicos, con una consolidación más temprana, apoyan mejores resultados con las placas bloqueadas. Esto se podría explicar por un montaje más estable, pero que a la vez comprime frente a la simple compresión de los tornillos canulados.

Aunque insuficiente para obtener conclusiones fiables, nuestro estudio sí parece mostrar una tendencia a mejores resultados clínicos en el grupo de placas bloqueadas frente a los tornillos canulados cruzados, que previamente no se había visto en la literatura actual. Sin embargo, los resultados mostrados de las dos técnicas en la evidencia actual son satisfactorios y fiables.

\section{Conclusión}

Hemos observado mejores resultados clínicos y radiológicos sin significación estadística utilizando la placa bloqueada.

Referencias

1. Stevens J, de Bot RT, Hermus J, van Rhijn L, Witlox A. Outcome following total joint replacement and arthrodesis for hallux rigidus: a systematic review. JBJS Rev. 2017; 5(11): e2.

2. Moon JL, McGlamry MC. First metatarsophalangeal joint arthrodesis: current fixation options. Clin Podiatr Med Surg. 2011; 28(2): 405-19.

3. Bauer T, Lortat-Jacob A, Hardy P. First metatarsophalangeal joint percutaneous arthrodesis. Orthop Traumatol Surg Res. 2010; 96(5): 567-73.

4. Treadwell JR. First metatarsophalangeal joint arthrodesis; what is the best fixation option? a critical review of the literature. Clin Podiatr Med Surg. 2013; 30(3): 327-49.
5. Harris E, Moroney P, Tourné Y. Arthrodesis of the first metatarsophalangeal joint: a biomechanical comparison of four fixation techniques. Foot Ankle Surg. 2017; 23(4): 268-74.

6. Claassen L, Plaass C, Pastor MF, Ettinger S, Wellmann M, StukenborgColsman C, et al. First metatarsophalangeal joint arthrodesis: a retrospective comparison of crossed-screws, locking and non-locking plate fixation with lag screw. Arch Bone Jt Surg. 2017; 5(4): 221-25.

7. Bei C, Gross C, Adams S, Parekh S. Dual plating with bone block arthrodesis of the first metatarsophalangeal joint: A clinical retrospective review. Foot Ankle Surg. 2015; 21(4): 235-9.

8. Asif M, Kassim S. Kannan S. A consecutive case series of 166 first metatarsophalangeal joint fusions using a combination of cup and cone reamers and crossed cannulated screws. J Foot Ankle Surg. 2018; 57(3): 462-5.

9. Donegan RJ, Blume PA. Functional results and patient satisfaction of first metatarsophalangeal joint arthrodesis using dual crossed screw fixation. J Foot Ankle Surg. 2017; 56(2): 291-7.

10. Dening J, van Erve RH. Arthrodesis of the first metatarsophalangeal joint: a retrospective analysis of plate versus screw fixation. $J$ Foot Ankle Surg. 2012; 51(2): 172-5.

11. Bass EJ, Sirikonda SP. 1st metatarsophalangeal joint fusion: a comparison of non-union and gender differences between locking and non-locking plating systems. Foot (Edinb). 2015; 25(4): 195-9.

12. Dalat F, Cottalorda F, Fessy MH, Besse JL. Does arthrodesis of the first metatarsophalangeal joint correct the intermetatarsal M1M2angle? Analysis of a continuous series of 208 arthrodeses fixed with plates. Orthop Traumatol Surg Res. 2015; 101(6): 709-14.

13. Wanivenhaus F, Espinosa N, Tscholl PM, Krause F, Wirth SH. Quality of early union after first metatarsophalangeal joint arthrodesis. $J$ Foot Ankle Surg. 2017; 56(1): 50-3.

14. Rammelt S, Panzner I, Mittlmeier T. Metatarsophalangeal joint fusion: why and how? Foot Ankle Clin. 2015; 20(3): 465-77.

Dirección para correspondencia:

Álvaro Martínez García

Dirección postal: c/Breton de los Herreros 56

2d Madrid, Madrid, 28003, España.

Tel: 0034699924636

E-mail: alvarommg1990@gmail.com

Conflicto de intereses: Los autores declaran que no presentan conflicto de intereses. 